\title{
Beyond 3G video mobile conversational services: An overview of 3G-324M based messaging and streaming
}

\author{
Andrea Basso \\ NMS Communications \\ andrea_basso@nmss.com
}

\author{
Hari Kalva \\ Florida Atlantic University \\ hari@cse.fau.edu
}

\begin{abstract}
$3 G-324 M$ is a $3^{\text {rd }}$ Generation Partnership Project (3GPP) umbrella standard for wireless video communications, which was created to satisfy the stringent requirements of real-time, low-delay interactive conversational video services. However it $i s$, in practice, employed today in $3 G$ networks to enable also a variety of multimedia services including messaging and streaming. The unification of this variety of services under the same umbrella standard has significance for the design of the supporting architectures and the applications that run such services. In this paper we will discuss the current limitations of 3G-324M in supporting streaming and messaging services and propose a set of requirements that imply some extensions and clarifications of the standard to better support them.
\end{abstract}

\section{Introduction}

Wireless carriers and application service providers are relying on $3 \mathrm{G}-324 \mathrm{M}$ for a variety of communication and entertainment service scenarios:

- Point-to-point video telephony.

- Wireless (TDM) to wireline (IP) video.

- Video enabled instant messaging and chat.

- Tromboning.

- Videoconferencing.

- Remote monitoring and surveillance.

- Video streaming.

- Video mail and video call answering.

- Multimedia real-time gaming.
In particular 3G-324M video streaming and video call answering are becoming part of several $3 \mathrm{G}$ carrier offerings today.

$3 \mathrm{G}-324 \mathrm{M}$ is a $3^{\text {rd }}$ Generation Partnership Project (3GPP) $[9,11,12,16,17]$ standard that enables multimedia conversational services over a wireless circuit-switched network. It comprises several protocol elements that handle a variety of functions including multiplexing and demultiplexing of speech, video, user, and control data (H.223) in a single $64 \mathrm{~Kb} / \mathrm{s}$ data circuit, in-band call control (H.245) video codecs (H.263 and MPEG-4) and audio codecs (AMR-NB and G.723.1).

3G-324M has several limitations in supporting video call answering and messaging.

As an example in 3G-324M video and audio channels are established at the beginning of the call and the media is coded in real-time. In addition the media characteristics are expected to remain relatively constant during the call. So there is no clear distinction between the definition of bitstream and channel because a single bitstream type is expected to flow in a given pre-established logical channel. The 3G-324M architecture has been designed for conversational services thus buffering is kept at a minimum in order to keep the delay low and allow interactivity. This limits the set of error concealment techniques that can be employed to improve the user experience.

On the contrary in video call answering, video messaging and video streaming the use of large buffers is allowed by the intrinsic nature of the services and, for a given session, the video messages left from various calles are coded either in different formats or with different flavors of the same format. Finally the modalities and the order with which these messages will played back depends on user preferences which are not known in advance. 
A naïve solution to these issues would be to allow video transcoding for every message so that the different video formats can be adapted to the format that was agreed at the beginning of the video call. Such solution is less that optimal for at least two reasons:

a) Inherent video quality degradation due to the transcoding process.

b) Much higher cost per channel due to the intense media processing required by transcoding.

In this paper we outline the current limitations of $3 \mathrm{G}$ $324 \mathrm{M}$ in support of services like streaming and video call answering/messaging and we propose a set of clarifications and extensions to the current specification that significantly improve the support of such services.

This paper is organized as follows: Section 2 briefly overviews the $3 \mathrm{G}-324 \mathrm{M}$ standard and its components. Section 3 discusses media adaptation and transcoding. Section 4 discusses some of the issues of 3G-324M video call answering and messaging services. Section 5 discusses some of the issues of $3 \mathrm{G}-324 \mathrm{M}$ streaming services. Section 6 presents a set of requirements for support of messaging and streaming in 3G-324M. Section 7 concludes the paper.

\section{Circuit-Switched Mobile Video: The 3G-324M Standard}

$3 \mathrm{G}-324 \mathrm{M}$ is an umbrella standard that enables realtime multimedia services over circuit-switched wireless networks. It includes protocol elements for multiplexing and demultiplexing of speech, video, user, and control data (H.223) in a single $64 \mathrm{~Kb} / \mathrm{s}$ circuit, call control (H.245) video (H.263 and MPEG-4) and audio codecs (AMR-NB and G.723.1). A block diagram of $3 \mathrm{G}-324 \mathrm{M}$ and the relevant $3 \mathrm{GPP}$ specifications is presented in Figure 1. Note that the 3GPP call setup requirements are spread in several documents [19-21].

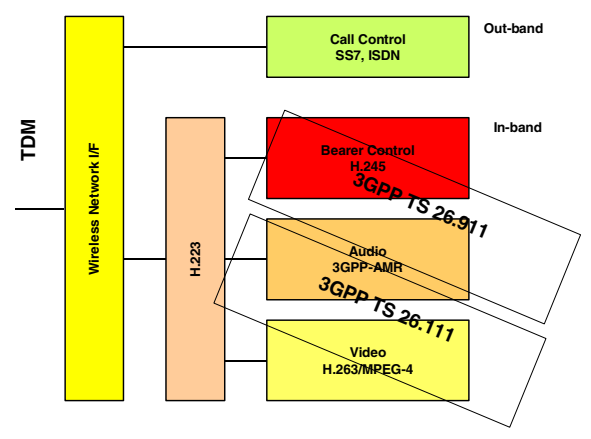

Figure 1: 3G-324M Components

\subsection{H.223 Multiplexing and Demultiplexing}

3G-324M defines three levels of H.223 [1] transport in order to provide different degrees of error-resilience.

Level 0, or baseline H.223, provides support for synchronization and bit stuffing. Level 0 allows 16 different multiplexing patterns to assemble media, control, and data packets. Multiplexing patterns are negotiated between the endpoints. The error resilience capabilities of Level 0 are limited. Bit errors can break the High-level Data Link Controller (HDLC) protocol, can interfere with bit stuffing, and can be the cause of flag emulations in the payload.

Level 1, defined in H.223 Annex A [2], provides a synchronization mechanism that considerably improves performance over error-prone channels. HDLC is replaced by a more robust framing and framing flag of larger length.

Level 2, defined by H.223 Annex B [3], is a further enhancement of Level 1, providing support for forward error correction (FEC) and including a larger header, which describes the contents of the multiplexed protocol data unit (PDU).

Level 3, defined by H. Annex C and D [4-5], is a further enhancement of Level 2, providing the most robust set of mobile extensions to the basic specification. This annex specifies both a new adaptation layer and a multiplexing layer and provide several enhancements including error detection and correction, sequence numbering, automatic repeat request and several retransmission schemes.

In H.223 every level comprises an adaptation, a multiplexing, and a demultiplexing layer. AL1, AL2, and AL3 are the three defined adaptation layers. AL1 is designed for data transfer and is typically used to transport user data and H.245 control messages. It relies on upper layers for error control and handling. AL2 provides an 8-bit cyclic redundancy check (CRC) and optional sequence numbering to allow loss detection. AL2 has the capability to handle AL service data units (SDUs) of variable length and is the preferred adaptation layer for the transport of audio data. AL3 is designed primarily for video and includes a 16-bit CRC and optional sequence numbering. It has the capability to handle AL SDUs of variable length and provides an optional retransmission procedure. 


\subsection{H.245 Call Control}

When parties start an H.245 conversation, it is necessary to decide which endpoint has the right to provide resolution in case of conflict. Thus H.245 defines a procedure to identify which party will be the master and which be slave during the H.245 conversation.

Endpoints may have different capabilities in terms of H.223 multiplexing/demultiplexing, supported video and speech codecs, data sharing, and other optional features. Thus H.245 provides a capability exchange functionality to allow the negotiation of such capabilities and to identify a set of features common to both endpoints.

The media and data flows are organized in logical channels. H.245 provides logical channel signaling to allow logical channel open/close and parameter exchange operations.

In H.245, the choice of codecs and their parameters are decided at the transmitter side on the basis of the capabilities that the receiver has advertised. If the receiver has a preference within its capability, it can signal it to the transmitter via a mode request.

Finally H.245 provides a variety of call control commands and indications to allow flow control, user input indications, video codec control, jitter indication, and skew. As an example H.245 "video fast update" command, also known as an "instantaneous decoder refresh", involves sending an intra picture to a decoder and thereafter refraining from using any picture sent prior to that intra picture as a reference for the decoding process of any subsequent picture sent in the stream. This command instructs the video encoder to complete the encoding of the current video picture and to generate a full intra picture at the earliest opportunity. The evaluation of such opportunity includes the current encoder coding strategy and the current available network resources. Intra pictures, independently from the instant in time when they are encoded, are in general several times larger in size than predicted pictures. Thus in scenarios in which the available bandwidth is small, the use of an intra picture implies a delay that is significantly longer than the typical picture duration.

H.245 User Input Indication (UII) plays a key role in all the services that require user interaction. In particular for video messaging, typical uses of UII include selection of user preferences, message recording and retrieval, and typical mailbox management functions. De-synchronization of media and control flows may affect the user experience (see section 6). H.245 is a reliable protocol, which ensures that the messages (i.e., DTMF tones) are guaranteed to be delivered and received at the far end. H.245 UII provides two level of representation of user indications: alphanumeric and the addition of duration information to the alphanumeric string to represent, for example, how long a given key has been pressed

H.245 [6] employs a Simple Retransmission Protocol (SRP) that can be optionally Numbered (NSRP) and provides a Control Channel Segmentation and Reassembly Layer (CCSRL) to ensure reliability in error-prone environments.

The use of SRP versus NSRP and CCSRL depends on the negotiated level during call setup. H.245 relies on Abstract Syntax Notation (ASN.1) for the definition of its messages. Furthermore the messages are binary encoded according to the Packed Encoding Rule (PER) [13].

\subsection{Speech Codecs}

The adaptive multi-rate (AMR) codec [17] is the mandatory speech codec in 3G-324M. G.723.1 [7] is an optional legacy codec included for backward compatibility with standards such as H.323. AMR can operate at different rates between 4.75 and $12.2 \mathrm{~kb} / \mathrm{s}$. It also supports comfort noise generation (CNG) and discontinuous transmission (DTX) mode. It can dynamically adjust its rate and error control, providing the best speech quality for the current channel conditions. The AMR codec also supports unequal error detection and protection (UED/UEP). This mode partitions the bit stream into classes on the basis of their perceptual relevance. An AMR frame is discarded if errors are detected in the most perceptually relevant data, otherwise it is decoded and error concealment is applied.

\subsection{Video Codecs}

3G-324M specifies H.263 [8] baseline level 10 as mandatory and MPEG-4 simple profile level 0 as recommended video codecs. H.263 has been kept for backward compatibility with standard as H.323. MPEG-4 [14] is more flexible than H.263 baseline and offers advanced error detection and correction schemes. Both codecs use Quarter Common Intermediate Format (QCIF) input picture format.

MPEG-4 provides a series of toolkits to boost error resilience. They include data partitioning, reversible 
variable length codes (RVLC), resynchronization markers, and header extension codes (HEC).

Data partitioning (DP) allows for syntactic boundaries to homogenous set of data. As an example data partitioning allows for the separation of Discrete Cosine Transform (DCT) coefficients and motion vector information by markers to prevent an error in one set from affecting the decoding of the other one. If errors are detected only in the DCT coefficients of a given macro-block, it will be possible to reconstruct the macro-block using the correct motion information while concealing the errors in the DCT coefficients. This results in a higher visual quality of the decoded picture than replacement of that macroblock with the corresponding one in the temporally previous frame.

RVLCs allow for the decoding of a given data block starting from its beginning (forward) or from its end (backward). This capability increases the chances of partial correct decoding of a set of corrupted data.

Resynchronization markers are codes inserted in the bit steam that allow the decoder to resynchronize the decoding process.

Header extension codes (HEC) allow a more effective resynchronization of the decoding process extending the resynchronization marker to also include timing information.

\section{Media Adaptation and Transcoding}

Multimedia mobile communications enabled by technologies such as $3 \mathrm{G}$, promise multimedia information access anytime, anywhere, on a variety of networked multimedia-enabled terminals. The problem, however, is how to provide content and services that are 'semantically equivalent' from a user perspective on such wide variety of terminals. The capabilities of these terminals differ in computing power, display sizes, network access, and supported network bandwidth. Media adaptation provides the framework to dynamically alter the content, e.g., picture size, encoding format, and content organization, while ensuring that the content semantics are kept as coherent and faithful as possible to the original. Adaptation is performed on the basis of terminal capabilities and user preferences. The media adaptation framework relies on several components:

- A multimedia information model for adaptation, which includes a hierarchy of content descriptors for the different modalities in which the multimedia content can be presented and delivered.

- A set of adaptation strategies that analyze the context information and compute and select the proper adaptation models.

- Media processing techniques for manipulating, translating, transcoding, and rearranging the multimedia content.

Standards such as MPEG-7 and more recently MPEG21 cover the definition of multimedia information models in support of media adaptation. Service providers typically define the viable adaptation strategies on the basis of the available media processing and delivery resources.

There are at least two classes of techniques to perform media adaptation - intra-media adaptation and crossmedia adaptation.

\subsection{Intra-media adaptation}

Intra-media adaptation relies on the characteristics of the particular media encoding scheme to perform the adaptation.

The adaptation operates on the video coding attributes, such as the video frame rate, the picture formats or the specific intra-frame and inter-frame quality, to meet a specific target size and format. Similarly, content can be transrated to meet specific terminal bandwidth constraints.

Transcoding is employed to adapt the content source to the different terminal coding standards. For applications and services based on 3G-324M, H.263/MPEG-4 transcoding is in general required because both video formats are part of the standard. Intra-media adaptation schemes imply in general a loss of perceptual quality. This is summarized in the case of transcoding in Figure 2. 


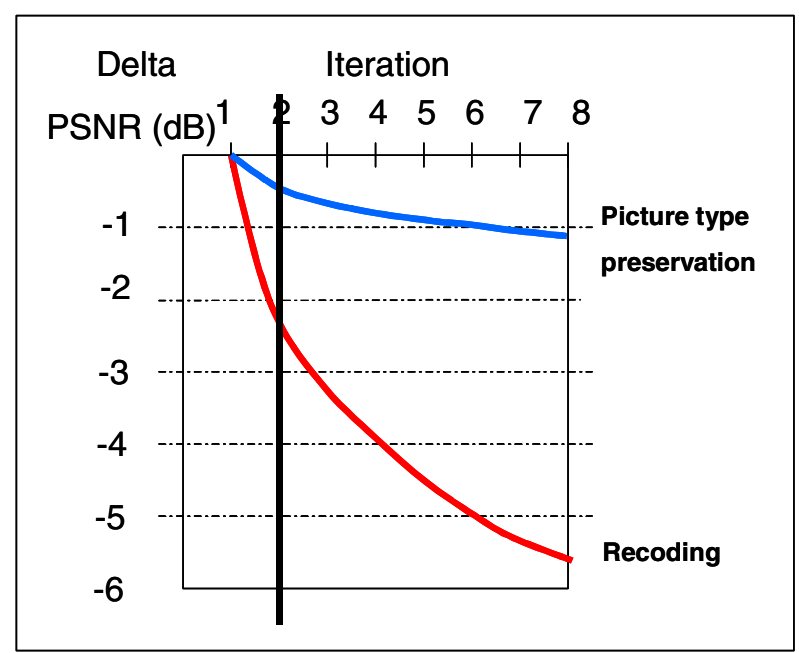

Figure 2: Video quality loss due to transcoding

10 test sequences including Bicycle, Mother and daughter, Miss America, Silent, Football, Mobile and Calendar, Flower Garden normalized to CIF format and 15 fps YUV 4:2:0 have been iteratively coded at $300 \mathrm{~Kb} / \mathrm{s}$ and decoded with the reference H.263 tmn320 [8] encoder and decoder and the Momusys reference MPEG-4 encoder and decoder [14]. H.263 was configured in a baseline mode and MPEG-4 was configured in a simple profile level 0 with RVLC enabled. On average a loss of $2 \mathrm{dBs}$ has been measured after the first transcoding iteration. Improvements can be obtained if more sophisticated transcoding techniques than simple picture recoding are used. Such techniques preserve as much as possible the decision modes that the original encoder selected for a given picture.

\subsection{Cross-media Adaptation}

It is possible to substitute a given media type and format with a "semantically equivalent" one, meaning that the substitution of media minimally affects the user-perceived content of the presentation. We will call this process cross-media adaptation. As an example, a given TV-format (720 x 480 pixels) video clip can be transformed into a sequence of "key" still pictures, sampled at scene changes or when considerable motion is detected in the scene, resized to QCIF format (176 x 144 pixels), synchronized with a lower rate coded version of the audio, and packaged as an MMS message that can be delivered to a $2.5 \mathrm{G}$ phone.

It is expected that the combination of both inter- and cross-media adaptation techniques will play an important role in the delivery of content in mobile video enabled services because the mobile environment has a very constrained presentation capability and limited network bandwidth.

\section{3G-324M video call answering and messaging.}

Figure 3 illustrates a typical 3G-324M video call answering scenario. If in case of no-answer call completion is handled by the $3 \mathrm{G}-324 \mathrm{M}$ video answering system by establishing a 3G-324M session with the caller. The caller may record a video message that can be played back by the callee at a later time. The same message can also in general be played back on a variety of video enabled terminals such as MMS $2.5 \mathrm{G}$ phones, set-top boxes, etc after the message has undergone a process of media adaptation.

\subsection{Message recording}

Message recording has no real-time requirements, thus H.223 selective retransmission techniques can be adopted in order to insure the best video quality and minimize the impairments and losses introduced by the wireless network.

In general the coding characteristics of the video material can change from message to message and depends on the video capabilities that have been negotiated during the recording session.

Message recording requires the video messaging system to request via a H.245 video fast update a reference picture so that the message can display properly at playback.

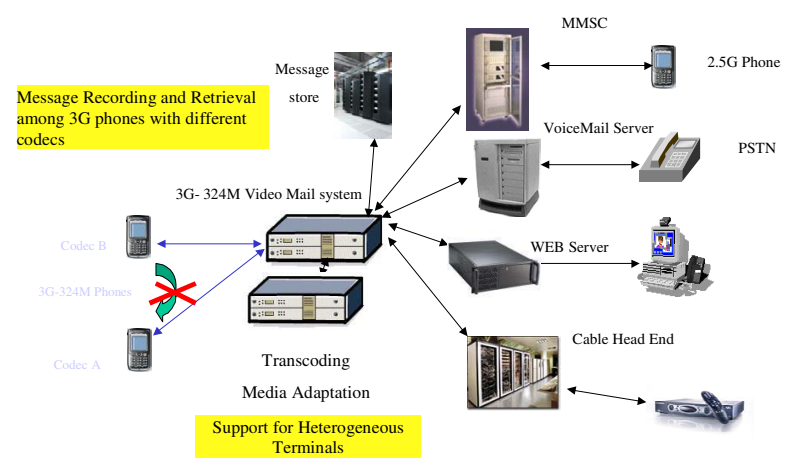

Figure 3: 3G-324M video call answering 


\subsection{Message Playback}

In this scenario a user accesses its own video mailbox where video messages coming from different users have been stored. The video messages are typically stored in different coding formats i.e. H.263 and MPEG-4. For each format several coding flavors are possible i.e. H.263 baseline or profile 3 with different combinations of annexes. Similarly in MPEG-4 simple profile level 0 the bit streams can be coded with options such as RVLC data partitioning or HEC.

In general it is not known at call setup time which messages the user will want to play back. The video call answering system will then typically negotiate a preferred video codec in order to minimize the amount of transcoding needed on the basis of a priori preferred codecs.

In great part of the architectures a real-time video transcoder is required if the message is not available in the playback format that the video call answering system has negotiated with the terminal. Alternatively the messages can be all transcoded offline and stored in all the admissible formats e but this is not always a viable alternative due to the storage cost and video processing requirements.

\subsection{Bitstream concatenation}

Message retrieval implies that multiple bitstreams with different coding characteristics are played back during the same session. In general a system video prompt is played back at the beginning of the session. It is followed by user-selected messages. Prompts can be generated on the fly by concatenation of elementary video and audio bitstreams. Messages in different formats (i.e. H.263 and MPEG-4) will require real-time transcoding.

In-band signaling can be used to signal the receiver the change in decoder configuration for messages coded in the same format. In-band signaling is supported in H.263. H.263 is a picture based coding scheme where all the needed information is in the picture header. Furthermore H.245 standard encourages H.263 inband signaling whenever possible.

MPEG-4 on the contrary has several problems with inband signaling of changes in the decoder configuration. MPEG-4 decoder configuration information (Video Object and Video Object Layer) is delivered in a reliable fashion via $\mathrm{H} .245$ and receivers rely on such $\mathrm{VO}$ and VOL information and disregard the VO/VOL information delivered in-band. As a consequence when a bistream with a different VO/VOL is played back on the same logical channel and the receiver relies only on the VO/VOL delivered via H.245 at the beginning of the call, the receiver may be not be able to successfully play back the video clip.

The 3GPP specification is not very clear on this topic. The reference [10] states that "When opening a logical channel for MPEG-4 Visual, configuration information (Visual Object Sequence Header, Visual Object Header, and Video Object Layer Header) shall be sent in the decoderConfigurationInformation parameter. The same information shall also be sent in the MPEG4 video bitstream. If the operational mode of MPEG-4 Visual encoder needs to be changed, the existing MPEG-4 video logical channel shall be closed and H.245 [6] procedures for opening a new MPEG-4 video logical channel shall be started. The new operational mode shall be indicated in the parameters of the new logical channel."

But it is unclear what a "change in operational mode" means. In fact it is possible to generate two different $\mathrm{VO} / \mathrm{VOLs}$ that refer to the same decoder operational mode . Let's assume that we have 2 VOLs, VOL1 and VOL2. In VOL1 the flag vol_control_parameters is set to FALSE and thus all the fields following (as described in table 6.2 .3 of [14]) are not present. In VOL2 instead vol_control_parameters is set to TRUE and the vol_control_parameters are set to the default, generating a stream that is operationally identical to the one described in VOL1 but VOL1 has a different binary representation than VOL2. Similar examples can be generated in reference to video_signal_type, aspect_ratio_info and others.

\subsection{Discontinuity in timing information}

Another issue that arises when concatenating pre recorded H.263 bitstreams is the discontinuity in the timing information. A discontinuity of the H.263 temporal Reference (TR) may cause a variable playback delay of several seconds.

\section{3G-324M-based Streaming}

A typical 3G-324M streaming scenario is presented in Figure 4. Media is retrieved via RTSP from a content server or live cameras. A 3G-324M /RTSP gateway maps signaling and media from the IP (RTP) side to the TDM side (3G-324M). 


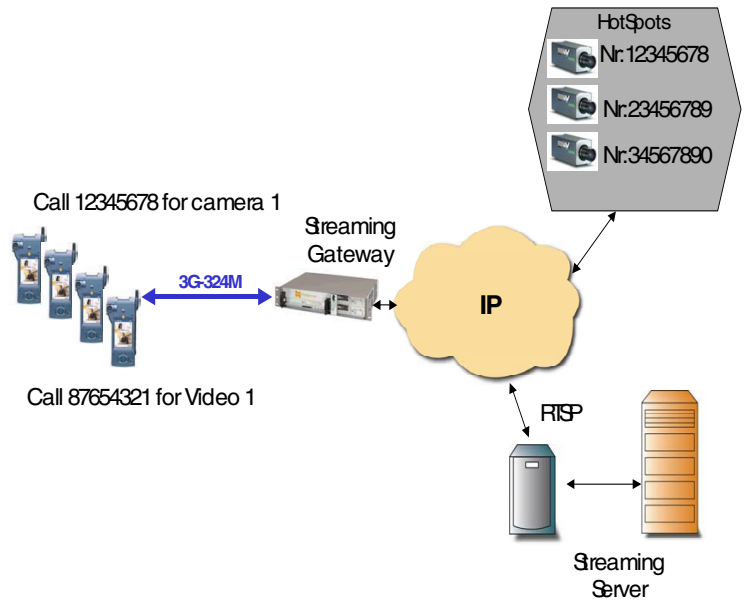

Figure 4: 3G-324M video streaming

The same issues that have been discussed for 3G-324M messaging playback are valid for the streaming scenario.

\section{Proposed 3G-324M Extensions and Clarifications.}

In this section a list of requirements that need to be addressed in $3 \mathrm{G}-324 \mathrm{M}$ is presented.

\subsection{User signaling support from endpoints to network device and vice versa.}

In a messaging or streaming system the user typically makes choices in selecting content, retrieving messages and managing messages (delete, skip, save, FF REW Stop, pause, etc) user profile, etc. The lack of signaling capability on the terminal will limit drastically the types of messaging and streaming services and services features. In the context of 3G-324M User Input Indication (UII) provides such functionality. In-band DTMF detection is discouraged because is unreliable due to the lossy AMR speech coding process.

A network device requires mechanisms to deliver reliably to the given endpoint a variety of information. Typical uses cases are network devices or streaming servers that need to provide to the endpoint information including status indication on length of the remaining playback, number of message left, text overlay on the video, etc.

\subsection{Temporal synchronization between the H.245 UII and the video stream.}

In general the video data has an inherent coding delay which can range from few tens to several hundreds of milliseconds. The H.245 UII message instead has negligible coding delay and gets delivered almost instantly. The de-synchronization between a command and the associated media on which the command is operating, may lead to an unacceptable user experience. As an example a desynchronized request to stop recording will lead to a message shorter that expected. The coding delay is particularly noticeable in endpoints. Assuming that the audio component has negligible delay with respect to UII, the H.245 message H223 skew indication could be used to estimate the delay with respect to the video bitstream. Unfortunately H.223 skew indication is not a mandatory message and thus we cannot rely on it to guarantee the proper user experience.

Alternatively the introduction of an empirically estimated delay can assure that no content is lost, but will affect the user experience generating in general longer than expected messages.

Only the endpoint can guarantee the synchronization between the video bitstream and the UII delivery when video is involved in a $3 \mathrm{G}-324 \mathrm{M}$ conversation thus it is mandatory that this synchronization is provided from the end point.

\subsection{Temporal synchronization between End of Session message and the video stream.}

Similarly to the previous case, the End Session message has negligible coding delay with respect to the video stream. If a call hang-up occurs and a End Session message is sent, the associated video message will not be recorded in its full length.

Assuming that the audio component has negligible delay with respect to H.245 UII, H223 skew indication could be used to estimate the delay with respect to the video bitstream and thus the delay delivery of the End session message. Unfortunately H.223 skew indication is not a mandatory message and thus it is impossible to guarantee the proper level of service.

Alternatively the introduction of an empirically estimated delay at the endpoint can assure that no content is lost but it will cause longer than expected pauses before hang-up.

It is essential that the terminal insures that all the content has been delivered before generating End Session command. 


\subsection{Playback of concatenated heterogeneous bitstreams in the same coding format.}

As already discussed in Section 4 in order to perform streamlined and cost effective operations a 3G-324M messaging system shall have the capability to concatenate prompts and messages in the same coding format ( but with different coding attirbutes) on the fly without or only minimal pre-processing. The server will deliver out-of-band (H.245) and also on the inband signaling of bitstream characteristic available in the bitstream headers as well as the markers that delimit the start and the end of a given video sequence. The endpoint video decoder shall reset the decoder once it receives and video End of Sequence (H.263 and MPEG-4). The endpoint should implement a similar behaviour. The endpoint video decoder shall reset the decoder once it receives a video End of Sequence as specified in H.263 and MPEG-4 standards.

\subsection{Handling of early termination of the media.}

On the request of early termination of a call a network device should deliver the remaining video at the next frame boundaries. The network device should send after the last video frame an end of video sequence. An endpoint should be able to reset and resume smooth playback of the next bitstream after receiving the end of sequence. Typical use cases include scenarios where a user that is playing back a message or a given selected content should be able to interrupt the message at any point during the playback and the playback of the next message or the new content should occur smoothly without video impairment.

\subsection{Streamlined storage support.}

The media format and framing on the air link and the wire should be as close as possible to the storage format.. In particular for AMR AMR IF2 is used in $3 \mathrm{G}-324 \mathrm{M}$ on the circuit side and it requires reframing on IF1 when stored in .3GP format. IF1 and IF2 should be supported in $3 \mathrm{GP}$ storage.

\section{Conclusions}

In this paper we have discussed the current limitations of $3 \mathrm{G}-324 \mathrm{M}$ in supporting streaming and messaging services and we have proposed some extensions and clarifications to the standard to significantly improve the support of such services.

\section{References}

[1] ITU-T Recommendation H.223: "Multiplexing protocol for low bit rate multimedia communication".

[2] ITU-T Recommendation H.223 - Annex A: "Multiplexing protocol for low bit rate multimedia mobile communication over low error-prone channels".

[3] ITU-T Recommendation H.223 - Annex B: "Multiplexing protocol for low bit rate multimedia mobile communication over moderate error-prone channels".

[4] ITU-T Recommendation H.223 - Annex C: "Multiplexing protocol for low bit rate multimedia mobile communication over highly error-prone channels".

[5] ITU-T Recommendation H.223 - Annex D: "Optional multiplexing protocol for low bit rate multimedia mobile communication over highly error-prone channels".

[6] ITU-T Recommendation H.245: "Control protocol for multimedia communication".

[7] ITU-T Recommendation G.723.1: "Dual rate speech coder for multimedia communication transmitting at 5,3 and $6,3 \mathrm{kbit} / \mathrm{s} "$

[8] ITU-T Recommendation H.263: "Video coding for low bitrate communication".

[9] ITU-T Recommendation H.324: "Terminal for low bitrate multimedia communication".

[10] 3GPP TR 26.111 V5.0.0 (2002-06) "Codec for circuit switched multimedia telephony service; Modifications to H.324"

[11] 3GPP TS 26.110: "Codec for Circuit Switched Multimedia Telephony Service; General description".

[12] 3GPP TR 26.911: "Codec for circuit switched multimedia telephony service; terminal implementor's Guide (Release 4)".

[13] ITU-T Recommendation X.691: "Information Technology - ASN.1 Encoding Rules - Specification of Packed Encoding Rules (PER)".

[14] ISO/IEC 14496-2: "Information technology - Coding of audio-visual objects - Part 2: Visual".

[15] 3GPP TS 26.071: "General description".

[16] 3GPP TS 26.090: "Transcoding functions".

[17] 3GPP TS 26.073: "Adaptive Multi-Rate (AMR); ANSI C source code".

[18] 3GPP TS 24.008: "Mobile radio interface layer 3 specification; Core Network Protocols-Stage 3"

[19] 3GPP TS 27.001 V4.11.0 (2003-09) "General on Terminal Adaptation Functions for Mobile Terminals"

[20] 3GPP TS 29.007 - General requirements on interworking between the Public Land Mobile Network (PLMN) and the Integrated Services Digital Network (ISDN) or Public Switched Telephone Network (PSTN).

[21] 3GPP TS 23.108 - Mobile radio interface layer 3 specification core network protocols; Stage 2 (structured procedures) 